\title{
Pulmonary histoplasmosis in a immunocompetent patient: A case report and literature review
}

\author{
CUI ZHU ${ }^{1}$, GE WANG ${ }^{2}$, QIONG CHEN ${ }^{1}$, BIXIU HE $^{1}$ and LIJING WANG ${ }^{1}$ \\ ${ }^{1}$ Department of Gerontology, Xiangya Hospital, Central South University, Changsha, Hunan 410008; \\ ${ }^{2}$ Department of Information and Technology Services, Zhengzhou Maternal and Child Health Hospital, \\ Zhengzhou, Henan 450012, P.R. China
}

Received June 4, 2015; Accepted July 22, 2016

DOI: $10.3892 /$ etm.2016.3774

\begin{abstract}
In the present study, the case of a 54-year-old male patient diagnosed with pulmonary histoplasmosis is reported, with the aim to increase the understanding of the disease characteristics and thereby facilitate the diagnosis and treatment of pulmonary histoplasmosis. Clinical manifestations, diagnosis, treatment and clinical outcomes of the present case of pulmonary histoplasmosis were described. In addition, 76 histoplasmosis patients with complete clinical data were reviewed by searching the literature for relevant studies published during 1990 and 2015. The disease was mainly manifested as cough in the present case, while imaging examination detected a lump shadow in the right lung, accompanied by exudative lesions. Initially, the patient was suspected to have bacterial pneumonia, but subsequently the diagnosis of pulmonary histoplasmosis was confirmed by lung biopsy. The symptoms were alleviated following itraconazole treatment. The patient was physically stable and had no recurrence during the subsequent follow-up period. In conclusion, pulmonary histoplasmosis is characterized by non-specific clinical and imaging manifestations, and lung tissue biopsy or respiratory pathogen culture are regarded as the diagnostic gold standards. Individualized antifungal medication should be administered based upon the patients' situation in terms of dosage and duration.
\end{abstract}

\section{Introduction}

Primary pulmonary histoplasmosis is a lung disease caused by the dimorphic fungus Histoplasma capsulatum, with a worldwide incidence. It is endemic in certain areas of North America, and was first reported in China in 1958 (1). The

Correspondence to: Dr Lijing Wang, Department of Gerontology, Xiangya Hospital, Central South University, 87 Xiangya Road, Changsha, Hunan 410008, P.R. China

E-mail: lijingwang201501@sina.com

Key words: pulmonary histoplasmosis, case report, itraconazole, levofloxacin, literature review severity of this mycosis is dependent upon the number of inhaled fungal particles and the immune status of the host (2). The morbidity and mortality rates of pulmonary histoplasmosis are low among immunocompetent patients. However, the morbidity and mortality rates of this mycosis are greater among immunocompromised individuals. In China, pulmonary histoplasmosis is sporadic. By 2015, only 76 cases with complete clinical data had been reported in the literature (3-20). Clinical presentations may include protean symptoms of fevers, chills, malaise, weight loss, cough and wheezing, symptoms. These are non-specific symptoms that may also occur with multiple other diseases. The majority of affected individuals present clinically silent manifestations and display no apparent symptoms (3). Therefore, misdiagnosis of pulmonary histoplasmosis as other pulmonary diseases, including tuberculosis, is highly likely. Misdiagnosis may delay the treatment, and the disease is fatal if left untreated (4).

Therefore, the present study reports the case of a patient diagnosed with pulmonary histoplasmosis. The study aimed to increase the understanding on the disease characteristics in order to facilitate the diagnosis and treatment of pulmonary histoplasmosis. The patient provided written informed consent for this case report.

\section{Case report}

A 54-year-old male patient was admitted to the Xiangya Hospital (Changsha, China) in June 2011 with a persistent cough that had appeared 1 week earlier due to contracting a cold. No signs of fever, sweating, chest pain, sputum, hemoptysis or other discomfort were noted. Pulmonary computed tomography (CT) scans conducted 2 days prior to admission detected a lump with enhanced intensity in the upper right lung and a large mass of high intensity in the right middle lobe with a pattern of air bronchogram observed in the mass (Fig. 1). Based on the characteristics observed in Fig. 1, the patient was suspected of having an infectious lung disease. Physical examination indicated the following: Body temperature, $36.5^{\circ} \mathrm{C}$; heart rate, 76 beats $/ \mathrm{min}$; blood pressure, 140/80 mmHg. In addition, no superficial lymphadenopathy was noted, while bilateral symmetry was observed in thoracic cage. No abnormalities were identified in the bilateral lung on percussion. Laboratory examination was also performed 
and revealed the following results: White blood cell count, $9.4 \times 10^{9} / 1$ (normal range, $4-10 \times 10^{9} / 1$ ); red blood cell count, $4.5 \times 10^{12} / 1$ (normal range, 3.5-5.5 $\times 10^{12} / 1$ ); hemoglobin level, $134 \mathrm{~g} / 1$ (normal range, 110-150 g/l); blood platelet count, $237 \times 10^{9} / 1$ (normal range, $100-300 \times 10^{9} / 1$ ); an elevated erythrocyte sedimentation rate, $70 \mathrm{~mm} / 1$ (normal range, $<20 \mathrm{~mm} / \mathrm{l}$ ); and negativity for tuberculosis antibody. In addition, the result of a G-test (for the detection of $(1,3)-\beta$-D-glucan, a fungal cell wall components) was abnormal at $57.0 \mathrm{pg} / \mathrm{ml}$ (normal range, $<20 \mathrm{pg} / \mathrm{ml}$ ). Bronchoscopy conducted 5 days after admission detected signs of inflammation of the bronchi, particularly the presence of severe inflammation in the right middle lobe. Negative acid-fast staining of the bronchus secretion was observed. Pathological examination under bronchoscopy revealed chronic mucosal inflammation in the branches of the right middle lobe. In combination with clinical data, the patient was preliminarily diagnosed with bacterial pneumonia. This diagnosis was made on the basis that lung infection is most commonly bacterial, whereas fungal infections often occur in immunodeficient patients and the present patient was an immunocompetent individual. Furthermore, G-tests may produce false positive results..

The patient was treated with levofloxacin ( $0.6 \mathrm{~g}$ once daily, intravenously; Daiichi Sankyo, Tokyo, Japan) for 2 weeks, starting from day 1 after admission; however, the symptoms were not alleviated. Lung CT scans 12 days after admission revealed that the infectious lesions increased in number and in size, as shown in Fig. 2. CT-guided needle lung aspiration biopsy was then performed. Subsequent pathological examination revealed signs of granulomatous inflammation in the right middle lung, as shown in Fig. 3. Based on the findings of CT scans and hematoxylin-eosin staining, the patient was suspected with histoplasmosis. Subsequently, the patient was diagnosed with pulmonary histoplasmosis, which was confirmed by the aforementioned imaging and pathological findings.

Following diagnosis, the patient was treated with itraconazole (Janssen Pharmaceuticals, Inc., Titusville, NJ, USA) for 2 weeks, starting from 15 days after admission. The dosage was $200 \mathrm{mg}$, by intravenous infusion, twice daily for the first 2 days and $200 \mathrm{mg}$ once daily thereafter. Subsequent lung CT scans conducted 4 weeks after admission revealed that the pulmonary infection was alleviated (Fig. 4), and the symptom of cough disappeared; thus, the patient was discharged from hospital. Subsequent to discharge, the treatment was altered to oral administration of itraconazole only (200 mg, twice daily, orally).

A further three rounds of lung CT scans performed in September 2011, November 2011 and February 2012 demonstrated that the pulmonary lesions were gradually adsorbed, as illustrated in Fig. 5A-C, respectively. A second CT examination scan in February 2012, 10 days after the previous one, revealed that all the lesions in the lung had almost disappeared (Fig. 5D); therefore, oral use of itraconazole was discontinued.

\section{Discussion}

Primary pulmonary histoplasmosFis is a primary lung disease caused by infection with fungus Histoplasma capsulatum.
Histoplasma capsulatum is a thermally dimorphic fungus, found in soil, caves and abandoned constructions that are enriched in bat or bird excrement. .Infection with Histoplasma capsulatum occurs by the inhalation of microconidia or mycelial fragments, which then settle in the host's lungs and convert to yeast forms. The invasion of this fungus is intimately associated with the host immunity. If the patient has strong cellular immunity, then macrophages, epithelial cells and lymphocytes can eliminate the fungi. However, in immunocompromised individuals, Histoplasma capsulatum disseminates to a variety of organs, including the bones, spleen, liver, adrenal glands and mucocutaneous membranes, resulting in progressive disseminated histoplasmosis (21).

In the present study, 'pulmonary histoplasmosis' was selected as the keyword to search the literature for relevant studies published between 1990 and 2015. The CNKI, VIP and Wanfang databases were searched for relevant studies. In addition, the PubMed database was searched with 'pulmonary histoplasmosis' and 'China' as search terms. Exclusion criteria were as follows: i) Cases who were repeatedly reported; ii) cases with pulmonary histoplasmosis evolving into disseminated histoplasmosis; and iii) cases with incomplete clinical data. In total, 18 eligible studies reporting 76 cases were identified and included for systematic review (3-20). Complete clinical data, including age, gender, risk factors, primary disease, clinical manifestation, misdiagnosis, confirmed diagnosis, treatment and prognosis were pooled and evaluated. Exclusion criteria were as follows: i) Cases who were repeatedly reported; ii) cases with pulmonary histoplasmosis evolving into disseminated histoplasmosis; and iii) cases with incomplete clinical data. Subsequent to case screening, a total of 76 patients, including 40 male and 36 female aged 27-69 years, were eventually recruited for subsequent analysis.

Among these patients, 52 cases were found to present increased risk factors or primary diseases, 5 cases were treated with glucocorticoid therapy or chemotherapy, and 1 case was orally treated with immunosuppressive agents for an immunodeficiency disease. In total, 46 patients were complicated with alternative diseases or high risk factors, including diabetes mellitus, viral hepatitis, tuberculosis, history of surgery and contact with poultry. However, the case reported in the present study had seldom contacted with duck, swine, cattle and other poultry, and had no history of any systemic disorders or use of immunosuppressive drugs prior to onset. The environment in which the present patient worked was not likely to have caused infection with Histoplasma capsulatum spore, suggesting that not all cases of pulmonary histoplasmosis have contact with the fungal spore or exposure to high risk factors.

Previous studies demonstrated that the symptoms of pulmonary histoplasmosis greatly vary, and $\sim 95 \%$ of patients diagnosed with pulmonary histoplasmosis present with atypical clinical manifestations (3-15). Common symptoms observed in the eligible studies mainly included fever in 11 cases, chest pain in 6 cases and other respiratory symptoms in 13 cases. However, in the current report, the patient solely presented with cough without apparent pulmonary infection.

The imaging manifestations of patients with pulmonary histoplasmosis are significantly diverse. Among the 77 cases, which included the 76 previous cases $(3-20)$ and the patient 
A

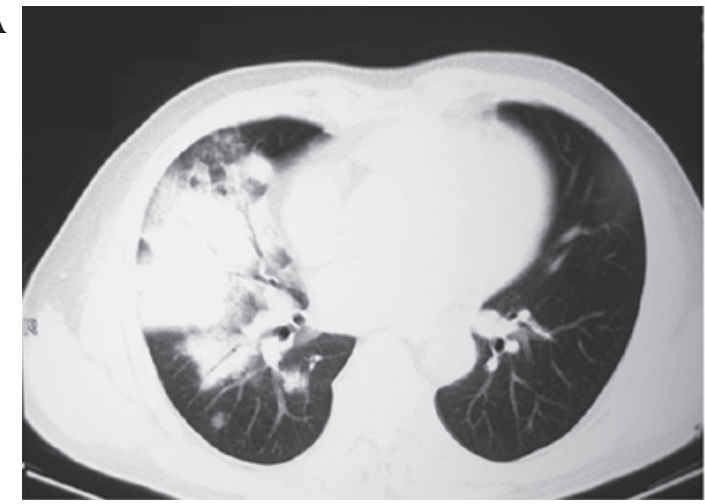

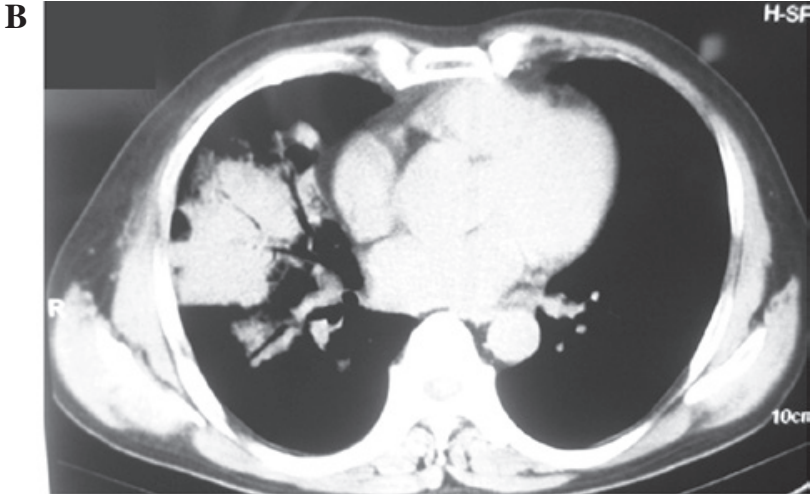

Figure 1. Computed tomography scans revealing bilateral pulmonary lesions 2 days before admission. (A) Pulmonary window and (B) mediastinal window.
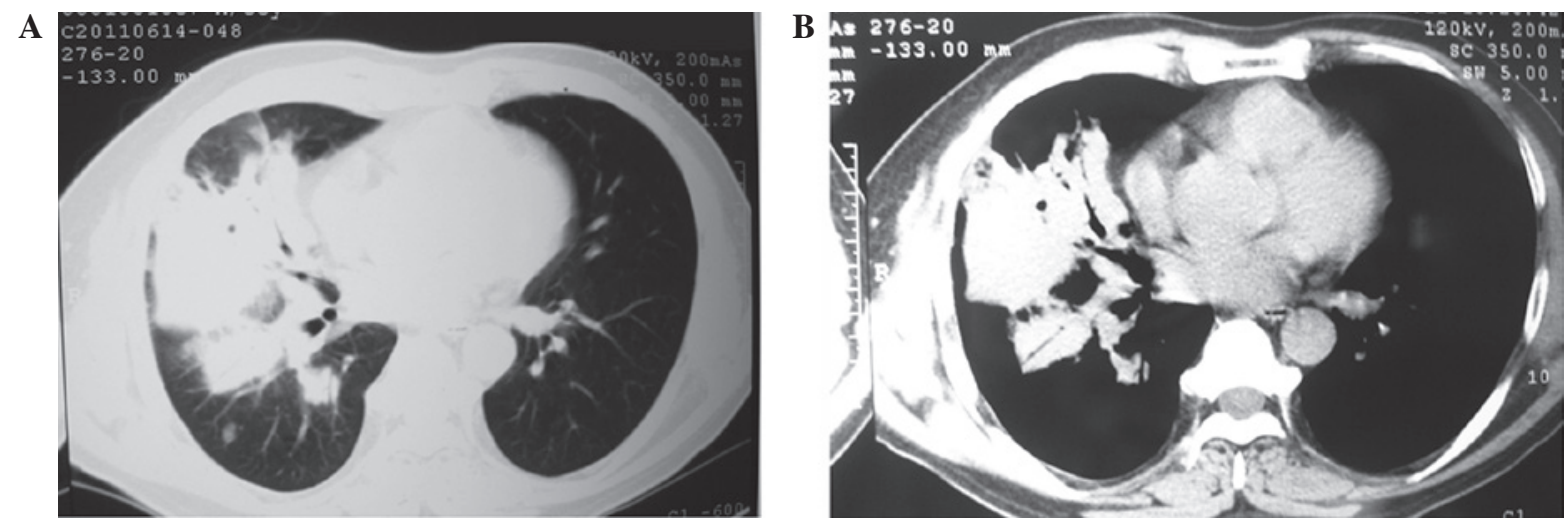

Figure 2. Computed tomography scans revealing the changes in the lung lesions subsequent to misdiagnosis and inappropriate therapy (12 days after admission). (A) Pulmonary window and (B) mediastinal window.

A

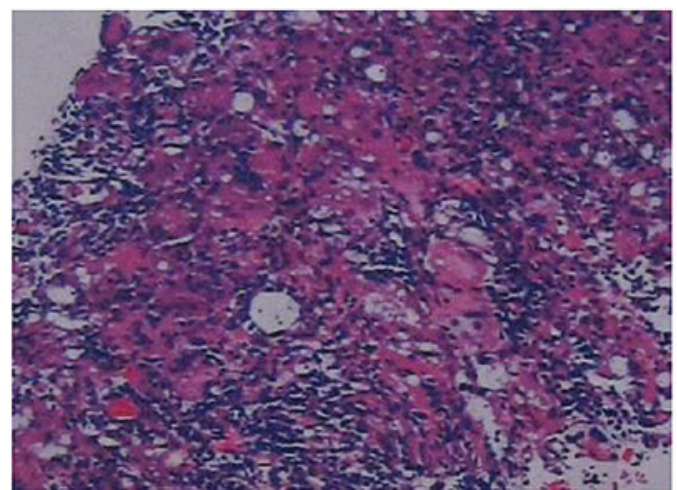

B

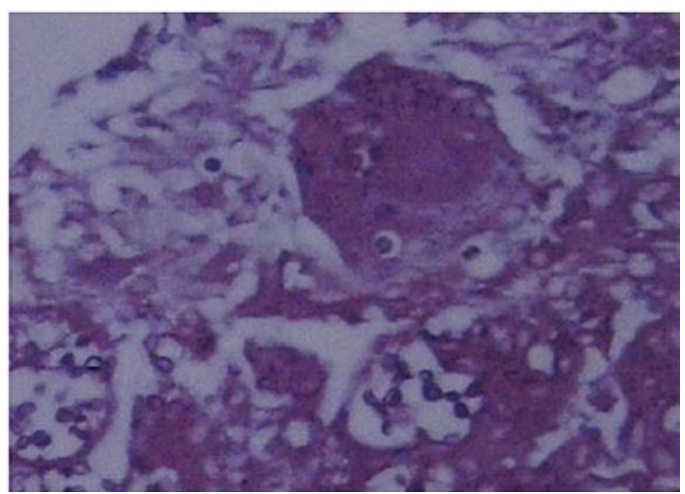

Figure 3. Hematoxylin and eosin staining revealing the changes in the pulmonary lesions. (A) Magnification, x200 and (B) magnification x400.

in the present study, nodular or lump lesions were noted in 50 patients $(64.9 \%)$, pneumonia-like exudative lesions were noted in 19 patients $(24.7 \%)$ and different shapes of lung lesions were detected in 8 patients (10.4\%) (22). Based on the findings of the present literature review, the following lung CT manifestations can be summarized for cases of pulmonary histoplasmosis: i) Cases with a short medical history of the disease are predominantly manifested as multiple sporadic exudative lesions of varying sizes and indistinct boundary. By contrast, cases with a long history of pulmonary histoplasmosis present with nodular lesions with an explicit boundary or Histoplasma capsulatum-infected tumors, or both; ii) sporadic or multiple lesions are frequently noted in the middle upper lobe of bilateral lungs; iii) pneumonia-like lesions, nodular lesions or both are observed; iv) exudates surround the pathological changes; v) the intensity enhancement is not evident; vi) pleural thickening is noted rather than pleural traction; and vii) the disease has a slow course. In the current study case report, the patient was found to have lump lesions in the right middle lung and exudates surrounding the lesions with insignificant enhancement. Initially, the present patient was misdiagnosed with bacterial pneumonia.

Lung tissue biopsy and fungal culture have been widely recognized as the gold standards for diagnosing pulmonary histoplasmosis $(23,24)$. Among the 77 cases enrolled in the present study, 74 cases were diagnosed by lung tissue biopsy, 
A

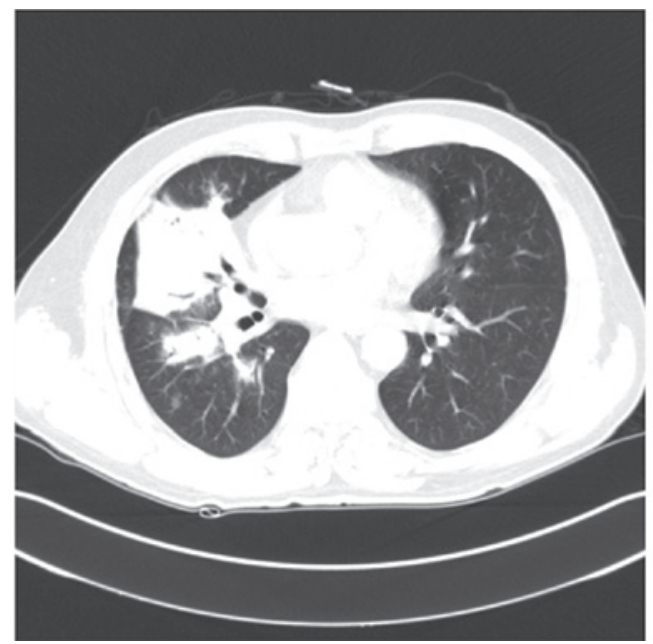

B

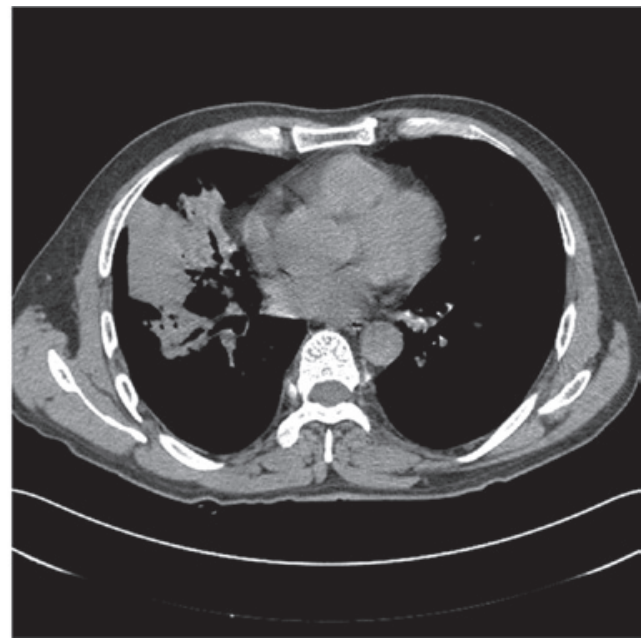

Figure 4. Computed tomography scans revealing the alleviation in the lung lesions subsequent to establishing the correct diagnosis and providing treatment (4 weeks after admission). (A) Pulmonary window and (B) mediastinal window.

A

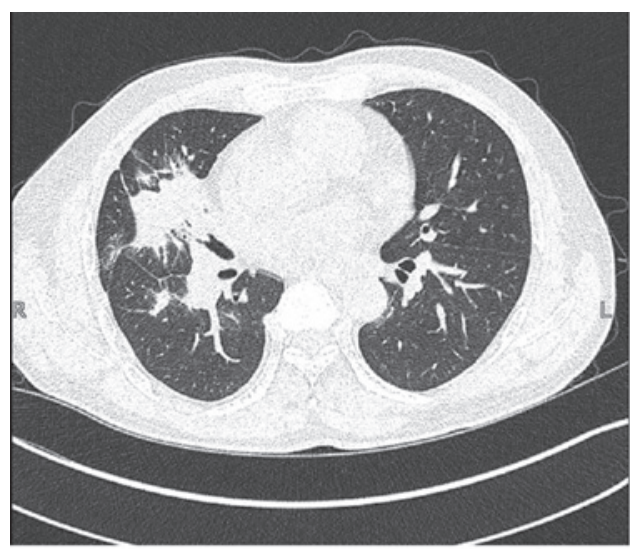

C

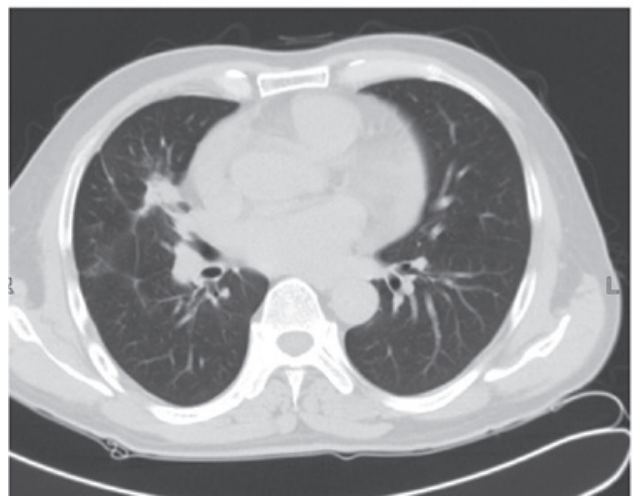

B

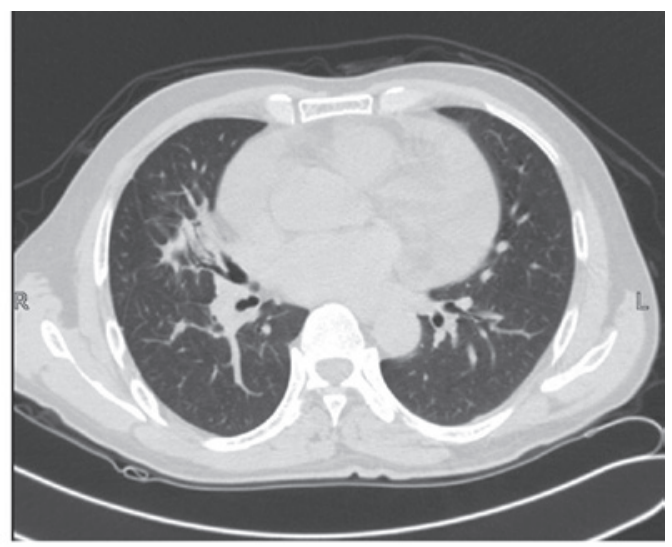

D

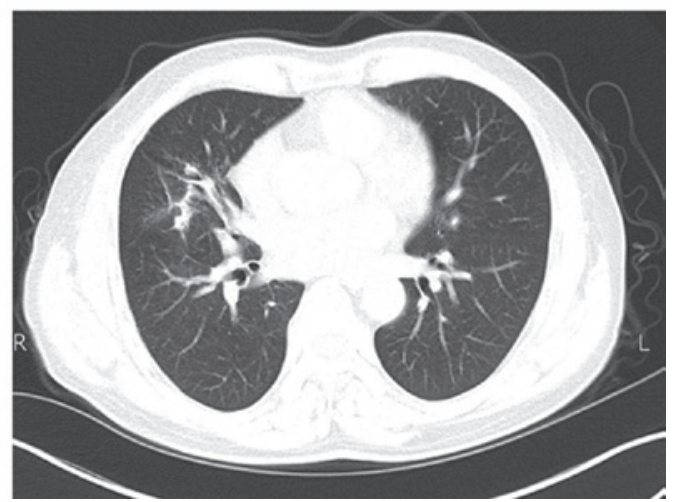

Figure 5. Computed tomography scans revealing the disappearance of the lung lesions following discharge. These scans were obtained in (A) September 2011, (B) November 2011, (C) February 2012 and (D) 10 days after the previous scan (February 2012).

while the remaining 3 cases were diagnosed by fungal culture. However, the fungal culture endured for at least 3 weeks and the positive rate was not adequately high; therefore, it has limited application in clinical practice (3-20). Similarly, the patient in the present case study was misdiagnosed with infectious pneumonia and showed no improvement following anti-infectious medication therapy. Subsequently, CT-guided percutaneous lung aspiration biopsy was conducted, which confirmed the diagnosis of pulmonary histoplasmosis. Consequently, bronchoscopy or CT-guided percutaneous lung aspiration biopsy is recommended to obtain histopathologic evidence and enhance the diagnostic accuracy when the diagnosis is ambiguous and undetermined.

According to the practice guidelines for histoplasmosis updated by the Infectious Diseases Society of America (25), different therapies should be delivered for acute or chronic pulmonary and disseminated histoplasmosis. Following literature analysis, 45 patients with histoplasmosis were surgically treated, 20 were treated with antifungal medication, 4 received itraconazole, 3 received triazole and 1 received with amphotericin B. In the present study, the patient fully recovered following the use of levofloxacin combined with 
itraconazole for 2 weeks during hospitalization and oral liquid of itraconazole for 10 months after discharge. Among the 77 cases, 38 patients were subject to follow-up for as long as $>20$ years. The majority of these patients were successfully treated or improved, whereas a minority of cases progressed into disseminated histoplasmosis or succumbed to the disease. In the current study, the patient was followed-up for $>10$ months and fully recovered following effective antifungal medication therapy.

In conclusion, the present study reported a case of pulmonary histoplasmosis that was initially misdiagnosed as bacterial pneumonia, and later successfully treated with antifungal therapy. For suspicious cases based on medical history and imaging manifestations, bronchoscopy or CT-guided lung needle aspiration biopsy should be actively performed to facilitate the differential diagnosis of pulmonary infection. After the diagnosis is confirmed, effective and proper antifungal treatment should be timely delivered upon the individual situations, aiming to enhance the clinical efficacy.

\section{References}

1. Li Y and Chen BQ: Histoplasmosis: One case report. Chin Med J 44: 301, 1958 (In Chinese).

2. Kauffman CA:Histoplasmosis: A clinical and laboratory update. Clin Microbiol Rev 20:115-132, 2007.

3. Wu XL, Shi YF and Wang HY: One case of pulmonary histoplasmosis. Zhejiang Lin Chuang Yi Xue 10: 105-106, 2008 (In Chinese).

4. Chen YB, Ji C, Ling CH, Huang J and Tao YD: Pulmonary histoplasmosis: One case report and literature analysis. Zhonghua Jie He He Hu Xi Za Zhi 31: 941-943, 2008 (In Chinese).

5. Zhu DQ, An SJ, Chen CJ and Wang SX: Two cases of pulmonary histoplasmosis. Linyi Yi Xue Zhuan Ke Xue Xiao Xue Bao 13: 257, 1991 (In Chinese).

6. Xie ZB, Zhong MH and Peng QZ: One case of pulmonary histoplasmosis. Lin Chuang Nei Ke Za Zhi 25: 702-703, 2008 (In Chinese).

7. Cai RZ, Li G and Cai YQ: Misdiagnosis of pulmonary histoplasmosis: One case report and literature review. Lin Chuang Hui Cui 27: 1912-1913, 2012 (In Chinese).

8. Wan P, Yan QH, Gao C, Xie YS, Yin QT, Li PL and Cao Y: Primary pulmonary histoplasmosis: One case report and literature review. Zhonghua Jie He He Hu Xi Za Zhi 17: 232-234, 1994 (In Chinese).

9. Li Q, Liu ZD, Huang Y and Hong XP: Chronic pulmonary histoplasmosis: One case report. Zhejiang Yi Xue 27: 448, 2005 (In Chinese).
10. Sun XY and Hong Z: Imaging manifestation of one case of pulmonary histoplasmosis. Zhonghua Fang She Xue Za Zhi 48: 782-783, 2014 (In Chinese).

11. Guo M, Zheng M, Xu Y and Chen Z: One case of pulmonary histoplasmosis suspected with lung cancer. Qiuyi Wenyao (Xueshuban) 9: 402-403, 2011 (In Chinese).

12. Ruan MJ, Zhu DZ, Wang XN and Wang JP: Pulmonary histoplasmosis with clinical characteristics similar to lung cancer. Zhong Guo Lin Chuang Yi Xue 9: 706-707, 2002 (In Chinese).

13. Zheng JX and Jia YM: Clinical analysis of four cases of reclusive pulmonary histoplasmosis. Zhenjiang Yi Xue Yuan Xue Bao 5: 303-304, 1995 (In Chinese).

14. Jiang CS, Luo XR, Qiu L, Zhang QH and Wang XS: One senior case of pulmonary histoplasmosis. Shi Yong Yi Xue Za Zhi 25: 301, 2009 (In Chinese).

15. Zhu ST: Pulmonary histoplasmosis with clinical characteristics similar to tuberculosis. Lin Chuang Wu Zhen Wu Zhi 14: 197, 2001 (In Chinese)

16. Gu YH, Zhou QY, Hou X, Xu YC, Tao JQ, Yang B, Yi J and $\mathrm{Xu}$ JR: Study on diagnosis and treatment of two cases of pulmonary infections caused by histoplasma. Zhonghua Yi Yuan Gan Ran Xue Za Zhi 24: 2477-2479, 2014 (In Chinese).

17. Wang LF, Guo L and Zhu M: CT features of primary pulmonary histoplasmosis. Lin Chuang Fang She Xue Za Zhi 31: 1727-1729, 2012 (In Chinese).

18. Zhang $\mathrm{F}$ and $\mathrm{Xu} \mathrm{Q}$ : One misdiagnosis case of pulmonary histoplasmosis. Zhong Guo Wu Zhen Xue Za Zhi 2: 1117, 2002 (In Chinese).

19. Shen G, Chai K, Zhang GF, Wei HQ and Yue L: Diagnosis and treatment of pulmonary histoplasmoma: Report of 3 cases. Zhonghua Yi Xue Za Zhi 87: 760-762, 2007 (In Chinese).

20. Xu C, Ma HT, Zhao J, Ni B and Li C: Surgical treatment of 22 cases with pulmonary histoplasmosis. Zhong Guo Wu Zhen Xue Za Zhi 10: 6005-6006, 2010 (In Chinese).

21. Garfoot AL, Zemska O and Rappleye CA: Histoplasma capsulatum depends on de novo vitamin biosynthesis for intraphagosomal proliferation. Infect Immun 82: 393-404, 2014.

22. Jin LL and Yang GZ: CT appearances of primary pulmonary histoplasmosis. Zhonghua Fang She Xue Za Zhi 43: 23-26, 2009 (In Chinese).

23. Cuellar-Rodriguez J, Avery RK, Lard M, Budev M, Gordon SM, Shrestha NK, van Duin D, Oethinger M and Mawhorter SD: Histoplasmosis in solid organ transplant recipients: 10 years of experience at a large transplant center in an endemic area. Clin Infect Dis 49: 710-716, 2009.

24. Grim SA, Proia L, Miller R, Alhyraba M, Costas-Chavarri A, Oberholzer J and Clark NM: A multicenter study of histoplasmosis and blastomycosis after solid organ transplantation. Transpl Infect Dis 14: 17-23, 2012.

25. Wheat LJ, Freifeld AG, Kleiman MB, Baddley JW, McKinsey DS, Loyd JE and Kauffman CA: Clinical practice guidelines for the management of patients with histoplasmosis: 2007 update by the infectious diseases society of America. Clin Infect Dis 45: 807-825, 2007. 